\title{
On homological stability for orthogonal and special orthogonal groups
}

\author{
Masayuki Nakada
}

\begin{abstract}
We shall prove that the $\operatorname{map} H_{i}\left(\mathrm{SO}_{n}(\mathbb{K}), \mathbb{Z}\right) \rightarrow H_{i}\left(\mathrm{SO}_{n+1}(\mathbb{K}), \mathbb{Z}\right)$ is bijective for $2 i<n$ and surjective for $2 i \leq n$. Here $\mathbb{K}$ is an arbitrary Pythagorean field and the special orthogonal group $\mathrm{SO}_{n}(\mathbb{K})$ is the subgroup of $\mathbb{K}$-linear automorphisms over $\mathbb{K}^{n}$ with determinant one which preserve the Euclidean quadratic form $\mathbf{q}(x)=x_{1}^{2}+\cdots+x_{n}^{2}$. It is derived from the homological stability of the orthogonal groups $\mathrm{O}_{n}(\mathbb{K})$ with twisted coefficients $\mathbb{Z}^{t}$.
\end{abstract}

\section{Introduction}

\section{1}

Let $\iota_{n}: G_{n} \rightarrow G_{n+1}(n \in \mathbb{N})$ be a sequence of groups, and let $\rho_{n}: M_{n} \rightarrow M_{n+1}$ $(n \in \mathbb{N})$ be a sequence of abelian groups where each $M_{n}$ is a $G_{n}$-module and $\rho_{n}$ is a $G_{n}$-module homomorphism through $\iota_{n}$. It defines a sequence of homomorphisms on homology groups of $G_{n}$ with coefficients in $M_{n}$ :

$$
\left(\iota_{n}\right)_{*}: H_{i}\left(G_{n}, M_{n}\right) \rightarrow H_{i}\left(G_{n+1}, M_{n+1}\right) .
$$

We say that a sequence of groups and modules $\left(G_{n}, M_{n}\right)$ satisfies the homological stability if for any $i$ there exists $n_{i}$ such that if $n>n_{i}$, then $\left(\iota_{n}\right)_{*}$ is an isomorphism. There are plenty of sequences of groups and modules which have the homological stability, and we are interested in the following cases.

Let $\mathrm{O}_{n}(\mathbb{K})$ be the orthogonal group over a field $\mathbb{K}$. It is the subgroup of linear transformations on $\mathbb{K}^{n}$ preserving the Euclidean quadratic form $\mathbf{q}(x)=$ $\sum x_{i}^{2}$ so that $\mathrm{O}_{n}(\mathbb{K})=\left\{x \in \mathrm{GL}_{n}(\mathbb{K}) \mid x^{t} x=E_{n}\right\}$. A quadratic space which is isometric to $\left(\mathbb{K}^{n}, \mathbf{q}\right)$ is called a Euclidean space. Now let $\mathbb{K}$ be a Pythagorean field, which means that the sum of two squares in $\mathbb{K}^{n}$ is always a square (see [4, Definition 8.3]), of characteristic different from 2. Quadratically closed fields and real-closed fields are typical examples of Pythagorean fields. In particular, the field of real numbers $\mathbb{R}$ and the field of complex numbers $\mathbb{C}$ are Pythagorean. Note that a field is Pythagorean if and only if every nondegenerate linear subspace of a Euclidean space is again Euclidean. Note also that, for any odd prime $p$ and any positive integer $f$, a finite field of $p^{f}$ elements has $\left(p^{f}+1\right) / 2$ squares. Since

Kyoto Journal of Mathematics, Vol. 55, No. 3 (2015), 627-640

DOI 10.1215/21562261-3089109, (C 2015 by Kyoto University

Received January 31, 2014. Revised July 11, 2014. Accepted July 28, 2014.

2010 Mathematics Subject Classification: $20 J 05$. 
$p$ is an odd prime, $\left(p^{f}+1\right) / 2$ does not divide $p^{f}$. This means that a Pythagorean field of characteristic different from 2 is never finite.

There is a standard inclusion $\iota_{n}: \mathrm{O}_{n}(\mathbb{K}) \rightarrow \mathrm{O}_{n+1}(\mathbb{K})$. We will let $\mathbb{Z}$ be the abelian group of integers with the trivial action. We denote by $H_{i}(G)$ the homology group with coefficients in $\mathbb{Z}$. Let $\mathbb{Z}^{t}$ be the abelian group of integers with the action through the determinant. This means that an element $g$ in $\mathrm{O}_{n}(\mathbb{K})$ acts on $n$ in $\mathbb{Z}$ as $(\operatorname{det} g) n$. Then $\left(\mathrm{O}_{n}(\mathbb{K}), \mathbb{Z}\right)$ and $\left(\mathrm{O}_{n}(\mathbb{K}), \mathbb{Z}^{t}\right)$ make sequences of groups and modules. The identity morphism on $\mathbb{Z}$ induces a sequence of homomorphisms on homology groups $H_{i}\left(\mathrm{O}_{n}(\mathbb{K})\right) \rightarrow H_{i}\left(\mathrm{O}_{n+1}(\mathbb{K})\right)$ and $H_{i}\left(\mathrm{O}_{n}(\mathbb{K}), \mathbb{Z}^{t}\right) \rightarrow$ $H_{i}\left(\mathrm{O}_{n+1}(\mathbb{K}), \mathbb{Z}^{t}\right)$. Let $\mathrm{SO}_{n}(\mathbb{K})$ denote the special orthogonal subgroup. If we restrict to $\mathrm{SO}_{n}$, then we get an isomorphism $\mathbb{Z}=\mathbb{Z}^{t}$ of $\mathrm{SO}_{n}$-modules. It defines a sequence of homomorphisms on homology groups $H_{i}\left(\mathrm{SO}_{n}(\mathbb{K})\right) \rightarrow H_{i}\left(\mathrm{SO}_{n+1}(\mathbb{K})\right)$.

We will prove that the following homological stability statements hold for any Pythagorean field $\mathbb{K}$ of characteristic different from 2 .

\section{THEOREM 1.1}

Let $\mathbb{K}$ be a Pythagorean field of characteristic different from 2. The induced maps on homology

$$
\left(\iota_{n}\right)_{*}: H_{i}\left(\mathrm{SO}_{n}(\mathbb{K}), \mathbb{Z}\right) \rightarrow H_{i}\left(\mathrm{SO}_{n+1}(\mathbb{K}), \mathbb{Z}\right)
$$

are bijective if $2 i<n$ and surjective if $2 i \leq n$.

\section{THEOREM 1.2}

Let $\mathbb{K}$ be a Pythagorean field of characteristic different from 2. The induced maps on homology

$$
\left(\iota_{n}\right)_{*}: H_{i}\left(\mathrm{O}_{n}(\mathbb{K}), \mathbb{Z}^{t}\right) \rightarrow H_{i}\left(\mathrm{O}_{n+1}(\mathbb{K}), \mathbb{Z}^{t}\right)
$$

are bijective if $2 i<n$ and surjective if $2 i \leq n$.

The theorems above extend and complement the following results, which are due to C. H. Sah and J.-L. Cathelineau.

\section{THEOREM 1.3}

(a) The induced maps

$$
\left(\iota_{n}\right)_{*}: H_{i}\left(\mathrm{O}_{n}(\mathbb{K})\right) \rightarrow H_{i}\left(\mathrm{O}_{n+1}(\mathbb{K})\right)
$$

are bijective if $i<n$ and surjective if $i \leq n$ (see [5], [2]).

(b) Let $\mathbb{Z}[1 / 2]$ be the ring of rational numbers whose denominators are powers of 2 . Then on homology with $\mathbb{Z}[1 / 2]$-coefficients, the induced maps

$$
\left(\iota_{n}\right)_{*}: H_{i}\left(\mathrm{SO}_{n}(\mathbb{K}), \mathbb{Z}[1 / 2]\right) \rightarrow H_{i}\left(\mathrm{SO}_{n+1}(\mathbb{K}), \mathbb{Z}[1 / 2]\right)
$$

are bijective if $2 i<n$ and surjective if $2 i \leq n$ (see [2]).

(c) The homology groups with twisted $\mathbb{Z}[1 / 2]$-coefficients $H_{i}\left(\mathrm{O}_{2 n}(\mathbb{K}), \mathbb{Z}[1 / 2]^{t}\right)$ are trivial if $i<n$ (see [2]). 
(d) For the field of real numbers $\mathbb{R}$,

$$
H_{2}\left(\mathrm{SO}_{3}(\mathbb{R})\right) \rightarrow H_{2}\left(\mathrm{SO}_{n}(\mathbb{R})\right) \rightarrow H_{2}\left(\mathrm{SO}_{n+1}(\mathbb{R})\right)
$$

are bijective if $n \geq 5$ (see [5]).

Cathelineau proved that the kernel of $\left(\iota_{n}\right)_{*}$ in $H_{n}\left(\mathrm{SO}_{2 n}(\mathbb{K}), \mathbb{Z}[1 / 2]\right)$ is equal to $H_{n}\left(\mathrm{O}_{2 n}(\mathbb{K}), \mathbb{Z}[1 / 2]^{t}\right)$, and if $\mathbb{K}$ is quadratically closed, then this kernel is the $n$th Milnor K-group of $\mathbb{K}$ tensored with $\mathbb{Z}[1 / 2]$, which is not zero in general (see [2, Theorem 1.5]). It is also conjectured that $H_{i}\left(\mathrm{O}_{2 n}(\mathbb{K}), \mathbb{Z}^{t}\right)$ is closely connected to motivic cohomology groups of $\mathbb{K}$ if $n<i<2 n$, which is supposed to be far from zero in general. We note also that these groups play an important role in the calculation of scissors congruence groups of spheres (see [3]).

We will see in the last section that $H_{i}\left(\mathrm{SO}_{n}\right) \rightarrow H_{i}\left(\mathrm{O}_{n}\right)$ are injective in the range of stability above.

\subsection{Notations}

A Pythagorean field $\mathbb{K}$ is fixed. Let us denote $\mathrm{O}_{n}(\mathbb{K})$ just by $\mathrm{O}_{n}$. We act similarly for $\mathrm{SO}_{n}$.

We use a standard isometric embedding of Euclidean spaces

$$
\mathbb{K}^{n} \rightarrow \mathbb{K}^{n+1}, \quad v \mapsto(0, v),
$$

which defines the inclusion map

$$
\iota_{n}: \mathrm{O}_{n} \rightarrow \mathrm{O}_{n+1}, \quad g \mapsto\left(\begin{array}{cc}
1 & 0 \\
0 & g
\end{array}\right)
$$

and its restriction between special orthogonal subgroups. Note that any other isometric embeddings are conjugate to the above one by the Witt extension theorem (see [4, p. 26]); hence, $\iota_{n}$ induces the same map in homology.

\section{Proofs of Theorems 1.2 and 1.1}

\subsection{Complex $C$}

An $l$-simplex is an ordered $(l+1)$-tuple of vectors $\left(v_{0}, \ldots, v_{l}\right)$ in $\mathbb{K}^{n+1}$. We assume that all $v_{i}$ 's are on $S\left(\mathbb{K}^{n+1}\right)=\left\{v \in \mathbb{K}^{n+1} \mid q(v)=1\right\}$. We call each $v_{i}$ a vertex of the simplex, and we call an ordered $(k+1)$-tuple $\left(w_{0}, \ldots, w_{k}\right)$ a face of the simplex if it is obtained from $\left(v_{0}, \ldots, v_{l}\right)$ by discarding some vertices. We say that an $l$-simplex is nondegenerate if the linear space spanned by all of its vertices is nondegenerate with respect to the quadratic form. An $l$-simplex $\left(v_{0}, \ldots, v_{l}\right)$ is called geometric if all of its faces are nondegenerate (see [2, Definition 2.1]).

In this paper we say that a geometric simplex $\left(v_{0}, \ldots, v_{l}\right)$ is normal if the set of vertices contains neither redundant pairs nor antipodal pairs. That is, for any different $i$ and $j, v_{i} \neq v_{j}$ and $v_{i} \neq-v_{j}$. Notice that every face of a normal simplex is again normal. Let $C_{l}$ denote the free $\mathbb{Z}$-module generated by normal $l$-simplices. We have that $\mathrm{O}_{n+1}$ acts diagonally on $l$-simplices:

$$
g \cdot\left(v_{0}, \ldots, v_{l}\right):=\left(g v_{0}, \ldots, g v_{l}\right)
$$


and this action sends any normal simplex to another normal simplex; hence, $C_{l}$ is an $\mathrm{O}_{n+1}$-module. We can define a homomorphism $\partial_{l}: C_{l} \rightarrow C_{l-1}$ as

$$
\partial_{l}\left(v_{0}, \ldots, v_{l}\right):=\sum_{i=0}^{l}(-1)^{i}\left(v_{0}, \ldots, \hat{v}_{i}, \ldots, v_{l}\right) .
$$

These define a chain complex of $\mathbb{Z} \mathrm{O}_{n+1}$-modules, and it has the augmentation homomorphism of the $\mathrm{O}_{n+1}$-module, where $a: C_{0} \rightarrow \mathbb{Z}$ is sending each 0 -vertex to 1 . Then

$$
0 \leftarrow \mathbb{Z} \stackrel{a}{\leftarrow} C_{0} \stackrel{\partial_{1}}{\longleftarrow} C_{1} \stackrel{\partial_{2}}{\longleftarrow} C_{2} \leftarrow \cdots
$$

is exact. This fact is derived from the extension property given in [2, Proposition 2.6(ii)] and [5]. Thus we get a resolution $C$. of $\mathbb{Z}$.

We set $C^{t} .=C . \otimes \mathbb{Z}^{t}$, and then $C^{t} \rightarrow \mathbb{Z}^{t}$ is a resolution. The associated spectral sequence (filtration by rows; see [6, Definition 5.6.2]) $E_{p, q}^{1}:=H_{p}\left(\mathrm{O}_{n+1}, C_{q}^{t}\right)$ strongly converges to $H_{p+q}\left(\mathrm{O}_{n+1}, \mathbb{Z}^{t}\right)$.

\section{2}

We have that $C$. is a subcomplex of the resolution associated with geometric simplices studied by Sah [5, Section 1]. We may use a variant of $C$. consisting of geometric simplices without having antipodal pairs of vertices. Then it would be a subcomplex of $C_{*}(n)$ in [2, Proposition 2.5] studied by Cathelineau.

\section{3}

There exists a filtration $\mathcal{F}^{s}$ of chain complexes of $\mathrm{O}_{n+1}$-modules on $C$. (see $[5$, Section 1], [2, Proposition 2.6]); $\mathcal{F}^{s}$ is generated by simplices $c$ having $\operatorname{dim}(c)$ less than or equal to $(s+1)$, where $\operatorname{dim}(c)$ is the dimension of the linear subspace in $\mathbb{K}^{n+1}$ spanned by the vertices of $c$. It is an increasing filtration of $\mathrm{O}_{n+1^{-}}$ modules on $C$., which induces a filtration $F_{p}^{\bullet}$ on $\left(E_{p,}^{1}, d^{1}\right)$ for each $p$ as $\left(F_{p}^{s}\right)_{q}=$ $H_{p}\left(\mathrm{O}_{n+1},\left(\mathcal{F}^{s}\right)_{q}\right)$.

\section{4}

We can choose a representative $\left(v_{0}, \ldots, v_{l}\right)$ in the $\mathrm{O}_{n+1}$-orbit of any simplex $c$ so that all the $v_{i}$ 's are in the $\mathbb{K}$-linear subspace spanned by the standard orthonormal bases $e_{1}, \ldots, e_{\operatorname{dim}(c)}$. We will write the orbit class which represents a simplex $\left(v_{0}, \ldots, v_{l}\right)$ as $\left[v_{0}, \ldots, v_{l}\right]$.

\section{5}

We will prove by induction on $n$ the following statement:

$$
\left(\iota_{n}\right)_{*}: H_{i}\left(\mathrm{O}_{n}, \mathbb{Z}^{t}\right) \rightarrow H_{i}\left(\mathrm{O}_{n+1}, \mathbb{Z}^{t}\right) \text { is } \begin{cases}\text { bijective } & \text { if } 2 i<n, \\ \text { surjective } & \text { if } 2 i \leq n .\end{cases}
$$

Note that if $n$ is an odd number $n=2 m+1$, then $\mathrm{O}_{2 m+1}$ contains a scalar matrix $-1_{2 m+1}$ of -1 , which has $\operatorname{det}\left(-1_{2 m+1}\right)=-1$. Therefore the center kills 
lemma (see [3, Lemma 5.4]) tells us that

$$
H_{i}\left(\mathrm{O}_{2 m+1}, \mathbb{Z}^{t}\right) \cong H_{i}\left(\mathrm{O}_{2 m+1}, \mathbb{Z}^{t}\right) \otimes \mathbb{Z} / 2
$$

for every $i$ and $m$. Thus, if $(2.1: n)$ is true, the following statement holds:

$$
\text { if } 2 i<n \text {, then } H_{i}\left(\mathrm{O}_{n}, \mathbb{Z}^{t}\right) \cong H_{i}\left(\mathrm{O}_{n}, \mathbb{Z}^{t}\right) \otimes \mathbb{Z} / 2 \text {. }
$$

Because $\mathrm{O}_{0}=\{1\}$ and $\mathrm{O}_{1}=\mathbb{Z} / 2$, the map

$$
\mathbb{Z}=H_{0}\left(\mathrm{O}_{0}, \mathbb{Z}^{t}\right) \stackrel{H_{0}\left(\iota_{0}, \mathbb{Z}^{t}\right)}{\longrightarrow} H_{0}\left(\mathrm{O}_{1}, \mathbb{Z}^{t}\right)=\mathbb{Z} / 2
$$

between coinvariant parts coincides with the epimorphism. We also have that

$$
\mathbb{Z} / 2=H_{0}\left(\mathrm{O}_{1}, \mathbb{Z}^{t}\right) \stackrel{H_{0}\left(\iota_{1}, \mathbb{Z}^{t}\right)}{\longrightarrow} H_{0}\left(\mathrm{O}_{2}, \mathbb{Z}^{t}\right)=\mathbb{Z} / 2
$$

is bijective; hence, (2.1:0) and (2.1:1) are true. We may assume that $n \geq 2$ from now on.

Firstly we have to show that

$$
E_{p, 0}^{1}=H_{p}\left(\mathrm{O}_{n+1}, C_{0}^{t}\right) \cong H_{p}\left(\mathrm{O}_{n}, \mathbb{Z}^{t}\right) \cong E_{p, 0}^{2} .
$$

From Shapiro's lemma (see [1, Proposition 6.2] or [3, Lemma 5.5]) we obtain that the first isomorphism $E_{p, 0}^{1} \cong H_{p}\left(\mathrm{O}_{n}, \mathbb{Z}^{t}\right)$ for the stabilizer subgroup of 0 simplex is isomorphic to $\mathrm{O}_{n}$. We have that

$$
E_{p, 1}^{1}=H_{p}\left(\mathrm{O}_{n+1}, C_{1}^{t}\right) \cong \bigoplus_{c} H_{p}\left(\operatorname{Stab}(c), \mathbb{Z}^{t}\right) \otimes \mathbb{Z} c
$$

where the index $c$ runs through all the $\mathrm{O}_{n+1}$-orbits of simplices in $C_{1}^{t}$, and $\operatorname{Stab}(c)$ is the stabilizer subgroup of $c$ in $\mathrm{O}_{n+1}$, where all the groups $\operatorname{Stab}(c)$ are isomorphic to $\mathrm{O}_{n-1}$ in this case.

Now let $c=\left(v_{0}, v_{1}\right)$ be a normal 1 -simplex, and let $\alpha$ be an element in $H_{p}\left(\operatorname{Stab}(c), \mathbb{Z}^{t}\right)$; then we have that

$$
d_{p, 1}^{1}\left(\alpha \otimes\left(v_{0}, v_{1}\right)\right)=\alpha \otimes\left(v_{1}\right)-\alpha \otimes\left(v_{0}\right) .
$$

We can find an element $g \in \mathrm{O}_{n+1}$ so that $g\left(v_{1}\right)=v_{0}$ and $\operatorname{det}(g)=1$ for $v_{0} \neq \pm v_{1}$ by the assumption of normality. Any such $g$ commutes with all the elements of $\operatorname{Stab}(c)$, and $g$ acts trivially on $H_{i}\left(\operatorname{Stab}(c), \mathbb{Z}^{t}\right)$; hence $\alpha \otimes\left(v_{1}\right)=\alpha \otimes g\left(v_{0}\right)=$ $\alpha \otimes\left(v_{0}\right)$ in $H_{i}\left(\operatorname{Stab}(c), \mathbb{Z}^{t}\right)$. This induces $d_{p, 1}^{1}(\alpha \otimes c)=0$. Thus, $d_{p, 1}^{1}=0$ on $E_{p, 1}^{1}$, which implies (2.4).

Secondly we have to show that

$$
E_{p, *}^{1} \text { is }(n-2 p-2) \text {-acyclic for } 0 \leq 2 p<n \text { augmented by } E_{p, 0}^{1}
$$

under the inductive hypothesis $\left(2.1: n^{\prime}\right)$ for all $n^{\prime}<n$.

If a geometric simplex $c$ has $\operatorname{dim}(c) \leq n-2 p$, then by the hypothesis of induction $(2.1: p)$, we get that

$$
H_{p}\left(\operatorname{Stab}(c), \mathbb{Z}^{t}\right) \cong H_{p}\left(\mathrm{O}_{n+1-\operatorname{dim}(c)}, \mathbb{Z}^{t}\right) \cong H_{p}\left(\mathrm{O}_{2 p+1}, \mathbb{Z}^{t}\right) .
$$


Thus, if $q \leq n-2 p-1$, then it holds that

$$
\begin{aligned}
E_{p, q}^{1} & =H_{p}\left(\mathrm{O}_{n+1}, C_{q}^{t}\right) \cong \bigoplus_{c} H_{p}\left(\operatorname{Stab}(c), \mathbb{Z}^{t}\right) \otimes \mathbb{Z} c \\
& \cong H_{p}\left(\mathrm{O}_{2 p+1}, \mathbb{Z}^{t}\right) \otimes \bigoplus_{c} \mathbb{Z} c .
\end{aligned}
$$

In particular, as we saw in (2.2) we have that the elements in (2.5) are annihilated by 2. (Notice that, through the isomorphism of Shapiro's lemma (2.5), $d_{p, *}^{1}$ may not equal $\mathrm{id}_{H_{p}\left(\mathrm{O}_{2 p+1}, \mathbb{Z}^{t}\right)} \otimes \partial_{*}$, because the action of $\mathrm{O}_{n+1}$ on $C$. is twisted in $H_{p}\left(\mathrm{O}_{n+1}, C_{q}^{t}\right)$ by the determinant and these data may cause a change of sign on the fixed representatives of $\mathrm{O}_{n+1}$-orbits. But this problem can be ignored because of (2.3) and the induction hypothesis in this case.)

We take $l$ arbitrarily for $0<l \leq n-2 p-2$. Let $\gamma \in E_{p, l}^{1}$ satisfy $d_{p, l}^{1}(\gamma)=0$. Apply (2.5), so

$$
\gamma=\sum_{j} \alpha_{j} \otimes\left[v_{0}^{j}, \ldots, v_{l}^{j}\right]
$$

where each $\alpha_{j}$ is in $H_{p}\left(\mathrm{O}_{2 p+1}, \mathbb{Z}^{t}\right)$ and $\left(v_{0}^{j}, \ldots, v_{l}^{j}\right) \in C_{l}$ is a representative chosen as in Section 2.4. Then we have $\operatorname{Span}_{\mathbb{K}}\left(v_{0}^{j}, \ldots, v_{l}^{j}\right) \perp e_{l+2}\left(\operatorname{Span}_{\mathbb{K}}\right.$ means the linear span of vectors), and the inclusion $\mathrm{O}_{2 p+1} \hookrightarrow \operatorname{Stab}\left(v_{0}^{j}, \ldots, v_{l}^{j}\right)$ factors through $\operatorname{Stab}\left(v_{0}^{j}, \ldots, v_{l}^{j}, e_{l+2}\right)$ for $l \leq n-2 p-2$. Since $\mathbb{K}$ is Pythagorean, $\left[v_{0}, \ldots, v_{l}, e_{l+2}\right]$ has a representative of geometric and thus normal simplex. Define $\gamma \# e$ as follows. For each orbit class of a normal $l$-simplex $\gamma=\left(v_{0}, \ldots, v_{l}\right)$, we set $\gamma \# e=$ $\left[v_{0}, \ldots, v_{l}, e_{l+2}\right]$. Then $\gamma \# e$ is normal and we extend this linearly: $\gamma \# e=\sum_{j} \alpha_{j} \otimes$ $\left[v_{0}^{j}, \ldots, v_{l}^{j}, e_{l+2}\right]$, which is contained in $E_{p, l+1}^{1}$. (This construction is called orthogonal join construction by Sah in [5, proof of (1.5)].) From Witt's extension theorem, we see that

$$
d_{p, l+1}^{1}(\gamma \# e)=d_{p, l}^{1}(\gamma) \# e+(-1)^{l+1} \gamma
$$

Since $d_{p, l}^{1}(\gamma)=0$, we obtain that $d_{p, l+1}^{1}(\gamma \# e)=(-1)^{l+1} \gamma$.

Finally we have to extend the acyclicity of $E_{p, *}^{1}$ one more degree above:

$$
E_{p, *}^{1} \text { is }(n-2 p-1) \text {-acyclic for } 0 \leq 2 p<n .
$$

Again we have that

$$
\begin{aligned}
E_{p, n-2 p}^{1} & =\bigoplus_{c} H_{p}\left(\operatorname{Stab}(c), \mathbb{Z}^{t}\right) \otimes \mathbb{Z} c \\
& =\bigoplus_{c} H_{p}\left(\mathrm{O}_{2 p}, \mathbb{Z}^{t}\right) \otimes \mathbb{Z} c \oplus \bigoplus_{c^{\prime}} H_{p}\left(\mathrm{O}_{2 p+1}, \mathbb{Z}^{t}\right) \otimes \mathbb{Z} c^{\prime},
\end{aligned}
$$

where the index $c$ in the first sum runs through $\mathrm{O}_{n+1}$-orbits of simplices in $C_{n-2 p}$ which satisfy $\operatorname{dim}(c)=n-2 p+1$, and the index $c^{\prime}$ in the second sum runs through $\mathrm{O}_{n+1}$-orbits of simplices which satisfy $\operatorname{dim}\left(c^{\prime}\right) \leq n-2 p$, that is, the second sum is in the associated filtration $F_{p}^{n-2 p-2}$. 
Let $\gamma \in E_{p, n-2 p-1}^{1}$ be such that $d_{p, n-2 p-1}^{1}(\gamma)=0$. If $\gamma \in F_{p}^{n-2 p-2}$, then the orthogonal join $\gamma \# e$ constructed as before is contained in the second component in (2.6), and it is a boundary element.

If $\gamma \notin F_{p}^{n-2 p-2}$, then we may assume that $\gamma$ is homologous to an element $\sum_{j} \alpha_{j} \otimes c_{j}$, where $\alpha_{j}$ is in $H_{p}\left(\mathrm{O}_{2 p+1}, \mathbb{Z}^{t}\right)$ and $c_{j}$ is an $\mathrm{O}_{n+1}$-orbit of an $(n-2 p-$ 1)-simplex. Since $\max \left\{\operatorname{dim}\left(c_{j}\right)\right\}=n-2 p$, we have that $\max \left\{\operatorname{dim}\left(c_{j} \# e_{n-2 p+1}\right)\right\}=$ $n-2 p+1$. The map $H_{p}\left(\mathrm{O}_{2 p}, \mathbb{Z}^{t}\right) \rightarrow H_{p}\left(\mathrm{O}_{2 p+1}, \mathbb{Z}^{t}\right)$ is surjective by the induction hypothesis $(2.1: p)$, so we can find $\beta_{j} \in H_{p}\left(\mathrm{O}_{2 p}, \mathbb{Z}^{t}\right)$ such that $H_{p}\left(\iota_{2 p}, \mathbb{Z}^{t}\right)\left(\beta_{j}\right)=\alpha_{j}$ for each $j$. Using these $\beta_{j}$ 's, we obtain that

$$
\begin{aligned}
& d_{p, n-2 p}^{1}\left(\sum_{j} \beta_{j} \otimes\left(c_{j} \# e_{n-2 p+1}\right)\right) \\
& \quad= \pm \sum_{j} \alpha_{j} \otimes\left(\partial c_{j}\right) \# e_{n-2 p+1}+(-1)^{n-2 p} \sum_{j} \alpha_{j} \otimes c_{j} \\
& \quad=\left(d_{p, n-2 p-1}^{1}(\gamma)\right) \# e+(-1)^{n-2 p} \gamma \\
& \quad=(-1)^{n-2 p} \gamma,
\end{aligned}
$$

and therefore we have proved that $\gamma$ is a boundary, which implies (2.6:p).

\section{6}

On the spectral sequence $E_{p, q}^{1}=H_{p}\left(\mathrm{O}_{n+1}, C_{q}^{t}\right) \Rightarrow H_{p+q}\left(\mathrm{O}_{n+1}, \mathbb{Z}^{t}\right)$, we know that, under the inductive assumption, $E_{p, 0}^{2} \cong H_{p}\left(\mathrm{O}_{n}, \mathbb{Z}^{t}\right.$ ) (see (2.4)) and $E_{p, q}^{2}=0$ for $0<q \leq n-2 p-1$ (see (2.6)). Therefore, the edge homomorphism coincides with the $\left(\iota_{n}\right)_{*}$ :

$$
\left(\iota_{n}\right)_{*}: H_{i}\left(\mathrm{O}_{n}, \mathbb{Z}^{t}\right) \rightarrow H_{i}\left(\mathrm{O}_{n+1}, \mathbb{Z}^{t}\right),
$$

which is bijective for $2 i<n$ and surjective for $2 i \leq n$. This ends the proof of Theorem 1.2.

\subsection{Bockstein exact sequences}

The group ring $\mathbb{Z}[\mathbb{Z} / 2]$ of $\mathbb{Z} / 2=\left\{\epsilon, \sigma \mid \sigma^{2}=\epsilon\right\}$ admits the action of $\mathrm{O}_{n}$ through the determinant:

$$
\begin{array}{lll}
g \cdot \epsilon=\epsilon, & g \cdot \sigma=\sigma & \text { if } \operatorname{det}(g)=1, \\
g \cdot \epsilon=\sigma, & g \cdot \sigma=\epsilon & \text { if } \operatorname{det}(g)=-1,
\end{array}
$$

for $g \in \mathrm{O}_{n}$. There exist an inclusion

$$
\mathbb{Z}^{t} \rightarrow \mathbb{Z}[\mathbb{Z} / 2], \quad 1 \mapsto \epsilon-\sigma
$$

and a projection

$$
\mathbb{Z}[\mathbb{Z} / 2] \rightarrow \mathbb{Z}[\mathbb{Z} / 2] /(\epsilon-\sigma) \cong \mathbb{Z}
$$

of (left) $\mathbb{Z} \mathrm{O}_{n}$-modules for $n \geq 0$. It makes a short exact sequence of $\mathbb{Z} \mathrm{O}_{n}$-modules

$$
0 \rightarrow \mathbb{Z}^{t} \rightarrow \mathbb{Z}[\mathbb{Z} / 2] \rightarrow \mathbb{Z} \rightarrow 0
$$


and we see that $H_{i}\left(\mathrm{O}_{n}, \mathbb{Z}[\mathbb{Z} / 2]\right) \cong H_{i}\left(\mathrm{SO}_{n}\right)$. (Use Shapiro's lemma and the fact that the stabilizer of $\mathrm{O}_{n}$ on $\mathbb{Z}[\mathbb{Z} / 2]$ is $\mathrm{SO}_{n}$.) We get a homology Bockstein exact sequence

$$
\cdots \rightarrow H_{i+1}\left(\mathrm{O}_{n}\right) \rightarrow H_{i}\left(\mathrm{O}_{n}, \mathbb{Z}^{t}\right) \rightarrow H_{i}\left(\mathrm{SO}_{n}\right) \rightarrow H_{i}\left(\mathrm{O}_{n}\right) \rightarrow H_{i-1}\left(\mathrm{O}_{n}, \mathbb{Z}^{t}\right) \rightarrow \cdots
$$

The inclusion $\iota_{n}: \mathrm{O}_{n} \rightarrow \mathrm{O}_{n+1}$ induces a homomorphism between exact sequences:

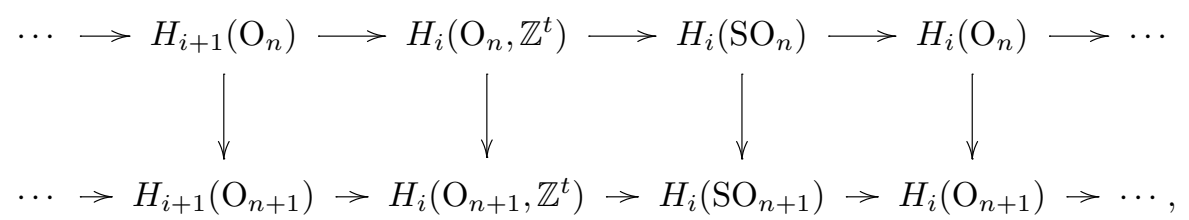

where columns are exact and maps in the vertical maps are induced from group inclusions $\iota_{n}: \mathrm{SO}_{n} \rightarrow \mathrm{SO}_{n+1}$ and $\iota_{n}: \mathrm{O}_{n} \rightarrow \mathrm{O}_{n+1}$. If we adapt Theorem 1.3(a) and $(2.1: n)$ in the above diagram, then, using the five lemma, we obtain Theorem 1.1.

\section{REMARK 2.1}

We have another short exact sequence

$$
0 \rightarrow \mathbb{Z} \rightarrow \mathbb{Z}[\mathbb{Z} / 2] \rightarrow \mathbb{Z}^{t} \rightarrow 0
$$

consisting of

$$
\mathbb{Z} \rightarrow \mathbb{Z}[\mathbb{Z} / 2], \quad 1 \mapsto \epsilon+\sigma
$$

and

$$
\mathbb{Z}[\mathbb{Z} / 2] \rightarrow \mathbb{Z}[\mathbb{Z} / 2] /(\epsilon+\sigma) \cong \mathbb{Z}^{t}
$$

\section{REMARK 2.2}

If we use the unmodified complex $\left(\mathcal{C}_{*}, \partial_{*}\right)$ used in [2, Proposition 2.5] (this may contain antipodal pairs but not contain simplices which have $v_{i-1}=v_{i}$ for some $i$ ), then

$$
\mathcal{E}_{p, 0}^{2}=H_{p}\left(\mathrm{O}_{n+1}, \mathcal{C}_{0}^{t}\right) \cong H_{p}\left(\mathrm{O}_{n}, \mathbb{Z}^{t}\right) \otimes \mathbb{Z} / 2 .
$$

This is because $\mathcal{C}_{1}$ admits the simplex $(v,-v)$ and the reflection that maps $v$ to $-v$ has determinant -1 . The spectral sequence defined by $\mathcal{E}_{p, q}^{1}=H_{p}\left(\mathrm{O}_{n+1}, \mathcal{C}_{q}^{t}\right)$ is also strongly convergent to $H_{p+q}\left(\mathrm{O}_{n+1}, \mathbb{Z}^{t}\right)$. Thus we can see that

$$
H_{i}\left(\iota_{n}, \mathbb{Z}^{t}\right): H_{i}\left(\mathrm{O}_{n}, \mathbb{Z}^{t}\right) \rightarrow H_{i}\left(\mathrm{O}_{n+1}, \mathbb{Z}^{t}\right)
$$

factors through $H_{i}\left(\mathrm{O}_{n}, \mathbb{Z}^{t}\right) \otimes \mathbb{Z} / 2$ for all $n$ and $i$. This implies that, though it is contained in an unstable range, $\operatorname{Im} H_{i}\left(\iota_{n}, \mathbb{Z}^{t}\right)$ is annihilated by 2 . 


\section{8. $\mathbb{Z} / 2$-coefficients}

We can improve the range of homological stability of special orthogonal groups with coefficients in $\mathbb{Z} / 2$. We use only Theorem 1.3 and the Bockstein exact sequence.

We have $(\mathbb{Z} / 2)^{t} \cong \mathbb{Z} / 2$ as $\mathrm{O}_{n}$-modules. Thus we have the same short exact sequence of $\mathrm{O}_{n}$-modules

$$
0 \rightarrow \mathbb{Z} / 2 \rightarrow \mathbb{Z} / 2[\mathbb{Z} / 2] \rightarrow \mathbb{Z} / 2 \rightarrow 0
$$

In the same way if we construct the Bockstein exact sequence from (2.8), then we get a long exact sequence

$$
\cdots \rightarrow H_{i+1}\left(\mathrm{O}_{n}, \mathbb{Z} / 2\right) \rightarrow H_{i}\left(\mathrm{O}_{n}, \mathbb{Z} / 2\right) \rightarrow H_{i}\left(\mathrm{SO}_{n}, \mathbb{Z} / 2\right) \rightarrow H_{i}\left(\mathrm{O}_{n}, \mathbb{Z} / 2\right) \rightarrow \cdots .
$$

From the universal coefficient theorem, Theorem 1.3(a) means that

$$
H_{i}\left(\mathrm{O}_{n}, \mathbb{Z} / 2\right) \rightarrow H_{i}\left(\mathrm{O}_{n+1}, \mathbb{Z} / 2\right) \text { is bijective for } i<n \text { and surjective for } i \leq n \text {. }
$$

Thus as in Section 2.7 we get the following result.

\section{PROPOSITION 2.3}

The map $H_{i}\left(\mathrm{SO}_{n}, \mathbb{Z} / 2\right) \rightarrow H_{i}\left(\mathrm{SO}_{n+1}, \mathbb{Z} / 2\right)$ is bijective for $i<n$ and surjective for $i \leq n$.

\section{Variants}

We consider a semidirect product of groups

$$
1 \rightarrow \mathrm{SO}_{n} \rightarrow \mathrm{O}_{n} \stackrel{\text { det }}{\longrightarrow} \mathbb{Z} / 2 \rightarrow 1 \quad \text { for } n \geq 1
$$

with a section

$$
s_{n}: \mathbb{Z} / 2 \rightarrow \mathrm{O}_{n}
$$

as $s_{n}(-1)=\operatorname{diag}(-1,1,1, \ldots, 1)$.

In the case $n=2 m+1$, it becomes the direct product of groups

$$
\mathrm{O}_{2 m+1} \cong \mathrm{SO}_{2 m+1} \times \mathbb{Z} / 2
$$

Thus there is the Künneth short exact sequence

$$
\oplus H_{p}\left(\mathrm{SO}_{2 m+1}\right) \otimes H_{q}(\mathbb{Z} / 2) \hookrightarrow H_{i}\left(\mathrm{O}_{2 m+1}\right) \rightarrow \oplus \operatorname{Tor}_{1}^{\mathbb{Z}}\left(H_{p}\left(\mathrm{SO}_{2 m+1}\right), H_{q}(\mathbb{Z} / 2)\right),
$$

and it is comparable with $\iota_{2 m+1}$. It is true that

$$
H_{i}\left(\mathrm{O}_{2 m+1}\right) \rightarrow H_{i}\left(\mathrm{O}_{2 m+3}\right)
$$

is bijective for $i<2 m+1$ and surjective for $i \leq 2 m+1$.

When $i \leq 2 m+1, \iota_{2 m+1}$ induces an isomorphism on the Tor terms by Section 1.1. We obtain the following result. 
PROPOSITION 3.1

We have that $H_{i}\left(\mathrm{SO}_{2 m+1}\right) \rightarrow H_{i}\left(\mathrm{SO}_{2 m+3}\right)$ is bijective for $i<2 m+1$ and surjective for $i \leq 2 m+1$.

On the other hand, (2.9) implies that

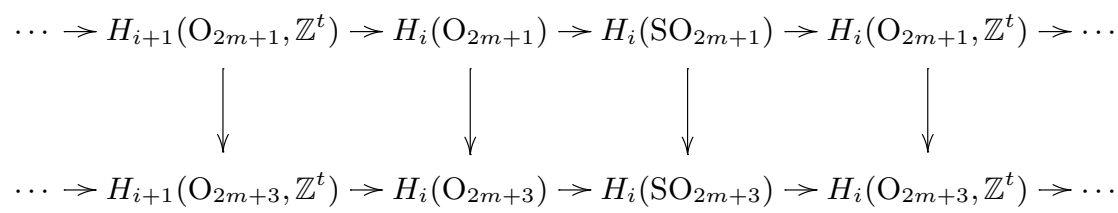

Thus we obtain that

$$
H_{i}\left(\mathrm{O}_{2 m+1}, \mathbb{Z}^{t}\right) \rightarrow H_{i}\left(\mathrm{O}_{2 m+3}, \mathbb{Z}^{t}\right)
$$

is bijective for $i<2 m+1$ and surjective for $i \leq 2 m+1$.

Notice that, using (2.1:n) and (3.6), we have that the sequence

$$
H_{i}\left(\mathrm{O}_{2 m-1}, \mathbb{Z}^{t}\right) \rightarrow H_{i}\left(\mathrm{O}_{2 m}, \mathbb{Z}^{t}\right) \rightarrow H_{i}\left(\mathrm{O}_{2 m+1}, \mathbb{Z}^{t}\right)
$$

splits as

$$
H_{i}\left(\mathrm{O}_{2 m}, \mathbb{Z}^{t}\right) \cong H_{i}\left(\mathrm{O}_{2 m-1}, \mathbb{Z}^{t}\right) \oplus K_{m, i}
$$

for $i<2 m$, where $K_{m, i}=\operatorname{Ker}\left\{H_{i}\left(\mathrm{O}_{2 m}, \mathbb{Z}^{t}\right) \rightarrow H_{i}\left(\mathrm{O}_{2 m+1}, \mathbb{Z}^{t}\right)\right\}$.

As we mentioned in $(2.2)$, we have that $H_{i}\left(\mathrm{O}_{2 m+1}, \mathbb{Z}^{t}\right) \cong H_{i}\left(\mathrm{O}_{2 m+1}, \mathbb{Z}^{t}\right) \otimes$ $\mathbb{Z} / 2$. Thus we get that, for $m \leq i<2 m$,

$$
\begin{aligned}
H_{i}\left(\mathrm{O}_{2 m}, \mathbb{Z}^{t}\right) & \cong \operatorname{colim}_{n} H_{i}\left(\mathrm{O}_{n}, \mathbb{Z}^{t}\right) \oplus K_{m, i} \\
& \cong H_{i}\left(\mathrm{O}_{\infty}, \mathbb{Z}^{t}\right) \otimes \mathbb{Z} / 2 \oplus K_{m, i} .
\end{aligned}
$$

4. $(\mathbb{Z} / 2)$-action on $H_{*}\left(\mathrm{SO}_{n}\right)$

There is a $(\mathbb{Z} / 2)$-action on $H_{i}\left(\mathrm{SO}_{n}\right)$ induced from the group extension (3.1). Let $\sigma$ denote the involution induced by $\sigma \in \mathbb{Z} / 2=\{\epsilon, \sigma\}$. The structure of this involution is important to apply the homological result to the problem of scissors congruence.

\subsection{Involution $\sigma$} PROPOSITION 4.1

The involution $\sigma$ on $H_{i}\left(\mathrm{SO}_{n}\right)$ is trivial if $2 i<n$.

We can write the action of the involution $\sigma$ on the bar resolution of $H_{i}\left(\mathrm{SO}_{n}\right)$ (see [1, Chapter I, Section 5]) as

$$
\left[g_{1}|\cdots| g_{i}\right] \mapsto\left[s_{n}(-1) g_{1} s_{n}(-1)^{-1}|\cdots| s_{n}(-1) g_{i} s_{n}(-1)^{-1}\right]
$$


For convenience, we write $\iota_{n}(g)=(1, g)$ for $\iota_{n}: \mathrm{SO}_{n} \rightarrow \mathrm{SO}_{n+1}$, and let $g^{\sigma}$ denote the image $s_{n}(-1) g s_{n}(-1)^{-1}$. We get that

$$
\begin{aligned}
& \left(-1, s_{n}(-1)\right) \iota_{n}\left(g^{\sigma}\right)\left(-1, s_{n}(-1)\right)^{-1} \\
& \quad=\left(-1, s_{n}(-1)\right)\left(1, s_{n}(-1) g s_{n}(-1)^{-1}\right)\left(-1, s_{n}(-1)\right)^{-1} \\
& \quad=\left(-1,1_{n}\right)(1, g)\left(-1,1_{n}\right)^{-1}=(1, g) .
\end{aligned}
$$

Since $\left(-1, s_{n}(-1)\right)=\operatorname{diag}(-1,-1,1, \ldots, 1)$ is contained in $\mathrm{SO}_{n+1}, H_{i}\left(\iota_{n}\right) \circ \sigma=$ $H_{i}\left(\iota_{n}\right)$. We obtain the following lemma.

\section{LEMMA 4.2}

We have that $H_{i}\left(\iota_{n}\right): H_{i}\left(\mathrm{SO}_{n}\right) \rightarrow H_{i}\left(\mathrm{SO}_{n+1}\right)$ factors through the $\sigma$-coinvariant part $H_{i}\left(\mathrm{SO}_{n}\right)_{\sigma}$ :

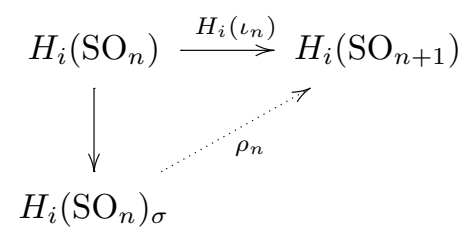

where the vertical map in the above diagram is the projection

$$
H_{i}\left(\mathrm{SO}_{n}\right) \rightarrow H_{i}\left(\mathrm{SO}_{n}\right) /(1-\sigma)=H_{i}\left(\mathrm{SO}_{n}\right)_{\sigma} .
$$

On the other hand, Theorem 1.1 claims that if $2 i<n$, then $H_{i}\left(\iota_{n}\right)$ must be an isomorphism; thus we have that

$$
H_{i}\left(\mathrm{SO}_{n}\right)^{\sigma} \cong H_{i}\left(\mathrm{SO}_{n}\right) \cong H_{i}\left(\mathrm{SO}_{n}\right)_{\sigma},
$$

and this implies Proposition 4.1.

\subsection{The edge homomorphism of the Lyndon-Hochschild-Serre spectral sequence}

The group extension (3.1) induces the Lyndon-Hochschild-Serre spectral sequence (see [1, Chapter VII, Theorem 6.8] or [6, Section 6.8])

$$
E_{p, q}^{2}=H_{p}\left(\mathbb{Z} / 2, H_{q}\left(\mathrm{SO}_{n}\right)\right) \Rightarrow H_{p+q}\left(\mathrm{O}_{n}\right)
$$

for $n \geq 0$. We will study the edge homomorphism

$$
e_{q}: H_{q}\left(\mathrm{SO}_{n}\right)_{\sigma}=E_{0, q}^{2} \rightarrow E_{0, q}^{\infty} \rightarrow H_{q}\left(\mathrm{O}_{n}\right) .
$$

\section{PROPOSITION 4.3}

We have that $e_{q}: H_{q}\left(\mathrm{SO}_{n}\right)_{\sigma} \rightarrow H_{q}\left(\mathrm{O}_{n}\right)$ is injective for $q \geq 0$.

\section{REMARK 4.4}

As we can see in [6, Section 6.8], $e_{q}$ is compatible with the map

$$
H_{i}(u): H_{i}\left(\mathrm{SO}_{n}\right) \rightarrow H_{i}\left(\mathrm{O}_{n}\right)
$$


induced by the natural inclusion $u: \mathrm{SO}_{n} \rightarrow \mathrm{O}_{n}$. We know that $H_{i}(u) \circ \sigma=H_{i}(u)$; thus, $H_{i}(u)$ factors through the $\sigma$-coinvariant part $H_{i}\left(\mathrm{SO}_{n}\right)_{\sigma}$, which is the edge homomorphism $e_{q}$.

The compositions with transfer maps

$$
H_{i}\left(\mathrm{SO}_{n}\right) \stackrel{H_{i}(u)}{\longrightarrow} H_{i}\left(\mathrm{O}_{n}\right) \stackrel{\operatorname{tr}}{\longrightarrow} H_{i}\left(\mathrm{SO}_{n}\right)
$$

and

$$
H_{i}\left(\mathrm{SO}_{n}\right) \stackrel{H_{i}\left(u, \mathbb{Z}^{t}\right)}{\longrightarrow} H_{i}\left(\mathrm{O}_{n}, \mathbb{Z}^{t}\right) \stackrel{\operatorname{tr}^{t}}{\longrightarrow} H_{i}\left(\mathrm{SO}_{n}\right)
$$

are the norm maps $(1+\sigma)$ and $(1-\sigma)$, respectively (see [1, Chapter III, Proposition 9.5]). Thus we have that

$$
\operatorname{Im}(\operatorname{tr}) \supseteq(1+\sigma) H_{i}\left(\mathrm{SO}_{n}\right)
$$

and

$$
\operatorname{Im}\left(\operatorname{tr}^{t}\right) \supseteq(1-\sigma) H_{i}\left(\mathrm{SO}_{n}\right),
$$

where the maps $\operatorname{tr}$ and $\operatorname{tr}^{t}$ are identified as

$$
H_{i}\left(\mathrm{O}_{n}\right) \rightarrow H_{i}\left(\mathrm{O}_{n}, \mathbb{Z}[\mathbb{Z} / 2]\right) \stackrel{\cong}{\rightarrow} H_{i}\left(\mathrm{SO}_{n}\right)
$$

and

$$
H_{i}\left(\mathrm{O}_{n}, \mathbb{Z}^{t}\right) \rightarrow H_{i}\left(\mathrm{O}_{n}, \mathbb{Z}[\mathbb{Z} / 2]\right) \stackrel{\cong}{\longrightarrow} H_{i}\left(\mathrm{SO}_{n}\right)
$$

in the Bockstein exact sequences (2.9) and (2.7), respectively. The map tr coincides with the trace map, and so does $\operatorname{tr}^{t}$ (see [1, Chapter III, Section 9]). Notice that the later map in (4.3) and (4.4) is an inverse of the map in Shapiro's lemma. It is induced from a map of chain complexes; namely,

$$
\rho:\left[g_{1}\left|g_{2}\right| \cdots \mid g_{i}\right] \otimes g \otimes x \mapsto\left[\widehat{g}^{-1} g_{1}{\widehat{z_{1}}}\left|{\widehat{z_{1}}}^{-1} g_{2} \widehat{z_{2}}\right| \cdots \mid{\widehat{z_{i-1}}}^{-1} g_{i} \widehat{z_{i}}\right] \otimes\left(\widehat{g}^{-1} g\right) x,
$$

where $\widehat{h}=\operatorname{diag}(\operatorname{det}(h), 1, \ldots, 1)$ and $z_{j}=g_{j}^{-1} \cdots g_{1}^{-1} g$, gives an isomorphism $H_{i}\left(\mathrm{O}_{n}, \mathbb{Z}\left[\mathrm{O}_{n}\right] \otimes_{\mathbb{Z}\left[\mathrm{SO}_{n}\right]} \mathbb{Z}\right) \cong H_{i}\left(\mathrm{O}_{n}, \mathbb{Z}[\mathbb{Z} / 2]\right) \cong H_{i}\left(\mathrm{SO}_{n}\right)$ (see [3, Remark after Lemma 5.5]). We can write the inverse direction

$$
H_{i}\left(\mathrm{O}_{n}\right) \rightarrow H_{i}\left(\mathrm{O}_{n}, \mathbb{Z}[\mathbb{Z} / 2]\right) \cong H_{i}\left(\mathrm{O}_{n}, \mathbb{Z} \mathrm{O}_{n} \otimes_{\mathbb{Z S O}} \mathbb{Z}\right)
$$

as

$$
\begin{aligned}
{\left[g_{1}|\cdots| g_{i}\right] } & \mapsto\left[g_{1}|\cdots| g_{i}\right] \otimes \epsilon+\left[g_{1}|\cdots| g_{i}\right] \otimes \sigma \\
& \mapsto\left[g_{1}|\cdots| g_{i}\right] \otimes 1_{n} \otimes 1+\left[g_{1}|\cdots| g_{i}\right] \otimes s_{n}(-1) \otimes 1
\end{aligned}
$$

hence, the composition with $\rho$ is

$$
\begin{aligned}
& {\left[{\widehat{1_{n}}}^{-1} g_{1}{\widehat{z_{1}}}\left|{\widehat{z_{1}}}^{-1} g_{2} \widehat{z_{2}}\right| \cdots \mid{\widehat{z_{i-1}}}^{-1} g_{i} \widehat{z_{i}}\right] \otimes\left({\widehat{1_{n}}}^{-1} 1_{n}\right) \cdot 1} \\
& \left.+\left[{\widehat{s_{n}(-1)}}^{-1} g_{1}{\widehat{z_{1}^{\prime}}}^{\mid z_{1}^{\prime}}{ }^{-1} g_{2}{\widehat{z_{2}^{\prime}}}^{\prime} \cdots \mid{\widehat{z_{i-1}^{\prime}}}^{-1} g_{i}{\widehat{z_{i}^{\prime}}}\right] \otimes{\widehat{s_{n}(-1)}}^{-1} s_{n}(-1)\right) \cdot 1 \\
& =\left[{\widehat{1_{n}}}^{-1} g_{1}{\widehat{z_{1}}}\left|{\widehat{z_{1}}}^{-1} g_{2}{\widehat{z_{2}}}\right| \cdots \mid{\widehat{z_{i-1}}}^{-1} g_{i}{\widehat{z_{i}}}\right] \otimes 1
\end{aligned}
$$




$$
\begin{aligned}
& +\left[s_{n}(-1)^{-1} g_{1} \widehat{z_{1}}\left|s_{n}(-1)^{-1}{\widehat{z_{1}}}^{-1} g_{2} \widehat{z_{2}} s_{n}(-1)\right|\right. \\
& \left.\cdots \mid s_{n}(-1)^{-1} \widehat{z_{i-1}}{ }^{-1} g_{i} \widehat{z}_{i} s_{n}(-1)\right] \otimes 1,
\end{aligned}
$$

where we set $z_{j}=g_{j}^{-1} \cdots g_{1}^{-1} 1_{n}$ and $z_{j}^{\prime}=g_{j}^{-1} \cdots g_{1}^{-1} s_{n}(-1)$. Now there is a chain homotopy (see [3, Lemma 5.4]) between

$$
\left[s_{n}(-1)^{-1} g_{1} \widehat{z_{1}}\left|s_{n}(-1)^{-1}{\widehat{z_{1}}}^{-1} g_{2} \widehat{z_{2}} s_{n}(-1)\right| \cdots \mid s_{n}(-1)^{-1} \widehat{z_{i-1}}{ }^{-1} g_{i} \widehat{z_{i}} s_{n}(-1)\right] \otimes 1
$$

and

$$
\sigma\left({\widehat{1_{n}}}^{-1} g_{1}{\widehat{z_{1}}}{\widehat{z_{1}}}^{-1} g_{2} \widehat{z_{2}}|\cdots|{\widehat{z_{i-1}}}^{-1} g_{i}{\widehat{z_{i}}}_{]} \otimes 1\right) .
$$

Thus, from the above calculation, we get that

$$
\operatorname{Im}(\operatorname{tr}) \subseteq(1+\sigma) H_{i}\left(\mathrm{SO}_{n}\right) .
$$

We can prove Proposition 4.3 by the diagram

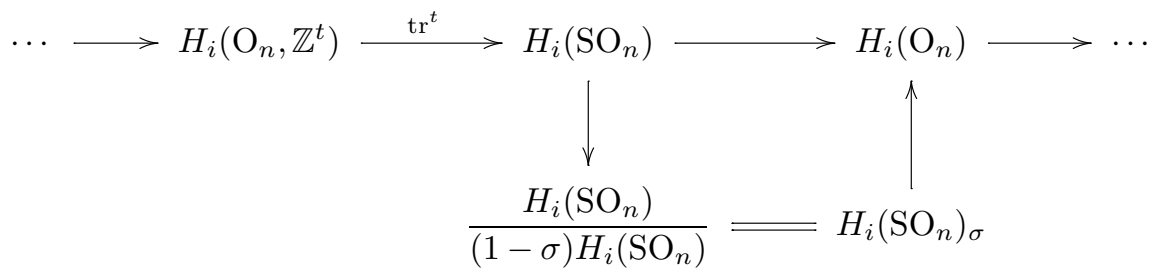

obtained by combining the exact sequence

$$
H_{i}\left(\mathrm{O}_{n}, \mathbb{Z}^{t}\right) \stackrel{\operatorname{tr}^{t}}{\longrightarrow} H_{i}\left(\mathrm{SO}_{n}\right) \rightarrow H_{i}\left(\mathrm{SO}_{n}\right) /(1-\sigma) H_{i}\left(\mathrm{SO}_{n}\right) \rightarrow 0
$$

and the Bockstein exact sequence (2.8).

In the same way, we can see that, in the Lyndon-Hochschild-Serre spectral sequence

$$
{ }^{t} E_{p, q}^{2}=H_{p}\left(\mathbb{Z} / 2, H_{q}\left(\mathrm{SO}_{n}\right)^{t}\right) \Rightarrow H_{p+q}\left(\mathrm{O}_{n}, \mathbb{Z}^{t}\right),
$$

the edge homomorphism

$$
{ }^{t} e_{q}: H_{q}\left(\mathrm{SO}_{n}\right)_{-\sigma} \rightarrow H_{q}\left(\mathrm{O}_{n}, \mathbb{Z}^{t}\right)
$$

is an injection.

\section{COROLLARY 4.5}

If $2 i<n$, then $\sigma$ on $H_{i}\left(\mathrm{SO}_{n}\right)$ is trivial as we saw in Proposition 4.1. Hence we obtain that $\operatorname{tr}^{t}: H_{i}\left(\mathrm{O}_{n}, \mathbb{Z}^{t}\right) \rightarrow H_{i}\left(\mathrm{SO}_{n}\right)$ is a zero map in this range.

Acknowledgments. The author would like to thank Prof. Masana Harada. He suggested that the author study group homology, and supported the author through the study of homological stability. He also cheered the author while he wrote this paper. The author would like to thank also Prof. Jean-L. Cathelineau and the anonymous referees for careful advice. 


\section{References}

[1] K. S. Brown, Cohomology of Groups, Grad. Texts in Math. 87, Springer, New York, 1982. MR 0672956.

[2] J.-L. Cathelineau, Homology stability for orthogonal groups over algebraically closed fields, Ann. Sci. École Norm. Supér. (4) 40 (2007), 487-517.

MR 2493389. DOI 10.1016/j.ansens.2007.03.001.

[3] J. L. Dupont, Scissors Congruence, Group Homology and Characteristic Classes, Nankai Tracts Math. 1, World Scientific, River Edge, N.J., 2001. MR 1832859. DOI 10.1142/9789812810335.

[4] T. Y. Lam, The Algebraic Theory of Quadratic Forms, W. A. Benjamin, Reading, Mass., 1973. MR 0396410.

[5] C.-H. Sah, Homology of classical lie groups made discrete, I: Stability theorems and Schur multipliers, Comment. Math. Helv. 61 (1986), 308-347. MR 0856093. DOI 10.1007/BF02621918.

[6] C. Weibel, An Introduction to Homological Algebra, Stud. Adv. Math. 38, Cambridge Univ. Press, Cambridge, 1994. MR 1269324. DOI 10.1017/CBO9781139644136.

Kobe University Secondary School, Kobe 658-0011, Japan;

mnakada@people.kobe-u.ac.jp 